\title{
Deep Learning Based Attenuation Correction of PET/MRI in Pediatric Brain Tumor Patients: Evaluation in a Clinical Setting
}

\author{
Claes Nøhr Ladefoged, Lisbeth Marner, Amalie Hindsholm, Ian Law, Liselotte Højgaard \\ and Flemming Littrup Andersen*
}

Department of Clinical Physiology, Nuclear Medicine and PET, Rigshospitalet, Copenhagen, Denmark

\section{OPEN ACCESS}

Edited by: Yangming $\mathrm{Ou}$ Harvard Medical School, United States

Reviewed by:

Yi Su,

Banner Alzheimer's Institute, United States

Sergey M. Plis,

The Mind Research Network (MRN), United States

*Correspondence: Flemming Littrup Andersen flemming.andersen@regionh.dk

Specialty section: This article was submitted to Brain Imaging Methods, a section of the journal Frontiers in Neuroscience

Received: 15 August 2018 Accepted: 13 December 2018 Published: 07 January 2019

Citation:

Ladefoged CN, Marner L, Hindsholm A, Law I, Højgaard L and Andersen FL (2019) Deep Learning Based Attenuation Correction of PET/MRI in Pediatric Brain Tumor

Patients: Evaluation in a Clinical Setting. Front. Neurosci. 12:1005 doi: 10.3389/fnins.2018.01005
Aim: Positron emission tomography (PET) imaging is a useful tool for assisting in correct differentiation of tumor progression from reactive changes. O-(2-18F-fluoroethyl)-Ltyrosine (FET)-PET in combination with MRI can add valuable information for clinical decision making. Acquiring FET-PET/MRI simultaneously allows for a one-stop-shop that limits the need for a second sedation or anesthesia as with PET and MRI in sequence. PET/MRI is challenged by lack of a direct measure of photon attenuation. Accepted solutions for attenuation correction (AC) might not be applicable to pediatrics. The aim of this study was to evaluate the use of the subject-specific MR-derived AC method RESOLUTE, modified to a pediatric cohort, against the performance of an MR-AC technique based on deep learning in a pediatric brain tumor cohort.

Methods: The modifications to RESOLUTE and the implementation of a deep learning method were performed using 79 pediatric patient examinations. We analyzed the 36 of these with active brain tumor area above $1 \mathrm{~mL}$. We measured background $(B)$, tumor mean and maximal activity ( $T_{\text {MEAN }}, T_{M A X}$ ), biological tumor volume (BTV), and calculated the clinical metrics $T_{M E A N} / B$ and $T_{M A X} / B$.

Results: Overall, we found both RESOLUTE and our DeepUTE methodologies to accurately reproduce the CT-AC clinical metrics. Regardless of age, both methods were able to obtain AC maps similar to the CT-AC, albeit with DeepUTE producing the most similar based on both quantitative metrics and visual inspection. In the patient-bypatient analysis DeepUTE was the only technique with all patients inside the predefined acceptable clinical limits. It also had a higher precision with relative \%-difference to the reference CT-AC (T $\mathrm{T}_{\mathrm{MAX}} / \mathrm{B}$ mean: $\left.-0.1 \%, \mathrm{Cl}:[-0.8 \%, 0.5 \%], p=0.54\right)$ compared to RESOLUTE (T MAX/B mean: $0.3 \%, \mathrm{Cl}:[-0.6 \%, 1.2 \%], p=0.67)$ and DIXON-AC (T MAX/B mean: 5.9\%, Cl: [4.5\%, 7.4\%], $p<0.0001)$.

Conclusion: Overall, we found DeepUTE to be the AC method that most robustly reproduced the CT-AC clinical metrics per se, closely followed by RESOLUTE modified to pediatric cohorts. The added accuracy due to better noise handling of DeepUTE, ease of use, as well as the improved runtime makes DeepUTE the method of choice for $\mathrm{PET} / \mathrm{MRI}$ attenuation correction.

Keywords: pediatric, deep learning, PET/MRI, attenuation correction, brain tumors, bone density, RESOLUTE 


\section{INTRODUCTION}

Positron emission tomography/Magnetic Resonance Imaging with the combination of MRI and radiolabeled amino acid analog tracers such as O-(2-18F-fluoroethyl)-L-tyrosine (FET) PET offer complimentary information when imaging cerebral brain tumors (Watanabe et al., 1992; Buchmann et al., 2016), especially when estimating the true tumor extent both in lowand high-grade gliomas (Kracht et al., 2004; Vander Borght et al., 2006). The combined information from the two modalities can help to discriminate post-operative changes or radiation damage from true tumor relapse presenting with a contrastenhanced region (Mullins et al., 2005; Vander Borght et al., 2006; Galldiks et al., 2015a,b). The experience with FET-PET in pediatric and adolescent patients is limited, but it has been shown that FET-PET can add valuable information for clinical decision making (Dunkl et al., 2015). For pediatric patients, there is a clear advantage of acquiring FET-PET simultaneously with conventional MRI, as it offers a one-stopshop examination, limiting the need for a second sedation or anesthesia as with PET and MRI in sequence, as well as improves co-registration (Henriksen et al., 2016). The advantage of a simultaneous PET/MRI comes with the challenge of accurate attenuation correction (AC) in order for the FET-PET images to be quantitatively correct (Vander Borght et al., 2006).

The initial shortcomings of the vendor-provided AC have been solved for examinations of adult brains without abnormal anatomy to a clinically acceptable precision (Ladefoged et al., 2016), whereas MR-based brain AC methods targeted toward pediatric subjects are scarce. Traditional atlas-based methods are likely to fail, since they are based on a database of adult subjects with normal anatomy (Spick et al., 2016). A database of pediatric age-matched subjects (Bezrukov et al., 2015) is difficult to obtain and might not be sufficient to model anatomical deformations following surgical intervention. An obvious alternative, the MRbased segmentation methods, is often challenged by the fact that traditional MR sequences are not able to distinguish bone and air due to the short relaxation time in bone. However, with special sequences such as ultra-short echo time (UTE) and zero echo time (ZTE), cortical bone can have a high signal despite its very short spin-spin relaxation time (Robson et al., 2003). Unfortunately, the use of these sequences is often hampered by incorrect representation of tissues at air/tissue interfaces (Ladefoged et al., 2015; Sekine et al., 2016) that needs to be specially addressed if a bias is to be avoided. We have recently introduced a PET/MRI-AC method, RESOLUTE (Ladefoged et al., 2015), that makes use of UTE images to calculate an attenuation map with continuous bone representation, and overcomes the air/tissue interface challenges by using anatomical regional masks defined on an aligned template in MNI space. Within these masks, possible bias from the bone surrogate signal is limited. We have shown that RESOLUTE led to the same clinical diagnosis as the reference CT-AC in a challenging cohort consisting of adult post-surgical brain tumor patients with severe anatomical deformations (Ladefoged et al., 2017). A prerequisite for successful application of RESOLUTE to pediatric cohorts is that these masks should be defined on pediatric templates.
Recently, deep learning using convolutional neural networks have demonstrated that they are able to handle complex signals, including noise, while maintaining a high level of accuracy (Han, 2017; Liu et al., 2017; Gong et al., 2018; Leynes et al., 2018). Using this technique, it could therefore be possible to limit the air/tissue interface noise without regional masks, thereby avoiding the need for any registration, as well as benefitting from the improved inference speed usually associated with deep learning. Several techniques using deep learning for MR-AC have been proposed (Han, 2017; Liu et al., 2017; Gong et al., 2018; Leynes et al., 2018), but none have been evaluated on a challenging cohort such as pediatrics.

The aim of this study was to modify the original RESOLUTE method to a pediatric cohort, and implement an MR-AC technique based on deep learning, that takes the UTE images as input and returns an attenuation map without any registration steps or need for regional masks. In a pediatric brain tumor cohort, we evaluated the attenuated FET-PET images of the modified RESOLUTE method, the proposed deep learning method and the vendor-provided DIXON-AC method using CT$\mathrm{AC}$ as reference standard, with the methods evaluated regionally, as well as with metrics used clinically for diagnosis and follow-up examinations.

\section{MATERIALS AND METHODS}

\section{Patients}

We included children with suspected brain tumor examined with FET-PET using our PET/MRI system (Siemens Biograph mMR, Siemens Healthcare, Erlangen, Germany) (Delso et al., 2011) between February 2015 and October 2017, and 86 FETPET examinations in total were identified of children under the age of 14 . Seven examinations were removed due to missing or corrupt data, resulting in 79 scans used to develop the method (average age: 8 years, min: 2 months, maximum 14 years). For evaluation of the four AC-methods, we included patients with an active tumor area above $1 \mathrm{~mL}$. Patients were part of a larger study of FET-PET/MRI in primary CNS tumors in children and adolescents approved by the regional ethical committee (ID: H-62014-095) and registered at clinicaltrials.gov (NCT03402425) and their parents gave written informed consent for participation.

\section{Acquisition of CT}

A reference low-dose CT image $(120 \mathrm{kVp}, 36 \mathrm{mAs}, 74$ slices, $0.6 \mathrm{~mm} \times 0.6 \mathrm{~mm} \times 3 \mathrm{~mm}$ voxels) of the head using a wholebody PET/CT system was used (Biograph TruePoint 40 and 64, Siemens Healthcare) (Jakoby et al., 2009). The CT images were acquired either on the same day as the PET/MRI examination, or at a previous $\mathrm{PET} / \mathrm{MRI}+\mathrm{CT}$ examination with no brain altering surgery in-between. The longest time for any patient between PET/MRI and low dose CT was 8 month.

\section{Acquisition of MRI}

The scan protocol included two vendor-provided AC methods: a two-point DIXON-VIBE AC sequence with repetition time (TR) $2,300 \mathrm{~ms}$, echo time 1 (TE1) $1.23 \mathrm{~ms}$, echo time 2 (TE2) $2.46 \mathrm{~ms}$, 
flip angle $10^{\circ}$, coronal orientation, $19 \mathrm{~s}$ acquisition time, voxel size of $2.6 \mathrm{~mm} \times 2.6 \mathrm{~mm} \times 3.12 \mathrm{~mm}$, and a UTE AC sequence with TR/TE1/TE2 $=11.94 / 0.07 / 2.46 \mathrm{~ms}$, a flip angle of $10^{\circ}$, axial orientation, $100 \mathrm{~s}$ acquisition time, software version VB20P, field of view (FOV) of $300 \mathrm{~mm}^{2}$, reconstructed on $192 \times 192 \times 192$ matrices $(1.6 \mathrm{~mm} \times 1.6 \mathrm{~mm} \times 1.6 \mathrm{~mm}$ voxels $)$.

\section{Acquisition of FET-PET}

Patients were positioned head first with their arms down on the fully integrated PET/MRI system. Data were acquired for $40 \mathrm{~min}$ immediately following injection of $3 \mathrm{MBq} / \mathrm{kg}(86 \pm 37)$ MBq FET (Langen et al., 2006) over a single bed position of $25.8 \mathrm{~cm}$ covering the head and neck. For the purpose of this study, the summed PET data 20-40 min after injection from the PET/MRI acquisition were reconstructed offline (E7tools, Siemens Medical Solutions, Knoxville, TN, United States) using 3D Ordinary Poisson-Ordered Subset Expectation Maximization (OP-OSEM) with 4 iterations, 21 subsets, zoom 2.5 and $5 \mathrm{~mm}$ Gaussian post-filtering on $344 \times 344$ matrices $\left(0.8 \times 0.8 \times 2 \mathrm{~mm}^{3}\right.$ voxels) in line with the clinical protocol used at our institution. For all images, default random, scatter and dead time correction were applied.

\section{Attenuation Correction Methods}

Four methods for AC were applied to the data. First, the CT image was co-registered to the UTE TE2 image, and was used as our gold standard AC reference following conversion of Hounsfield Units as implemented on the Siemens PET/CT system. Second, vendor-provided MR-based attenuation map were derived using the DIXON VIBE sequence (Martinez-Möller et al., 2009). Third, our recently proposed AC method, RESOLUTE, was updated to process the pediatric cohort on two areas: (1) the regional masks were re-drawn on pediatric templates in MNI space (Fonov et al., 2011) spanning the ages: $0-2 \mathrm{~m},<1$ year, $1-2,2-4,4-8,8-$ 11 , and 11-14 years, and (2) the $R_{2}^{*}$-CT bone mapping was calculated for the pediatric patients by the use of a sigmoid fit rather than a polynomial (Juttukonda et al., 2015). RESOLUTE was derived for each pediatric patient, where we used 2-fold cross validation to ensure that the mapping was not performed on the same patients used to recalibrate the mapping. Lastly, we implemented an MR-AC method based on deep learning convolutional neural networks, denoted DeepUTE. The network was based on a modified version of the U-net architecture (Ronneberger et al., 2015; Çiçek et al., 2016), where the max pool operations were replaced with convolutions with stride 2 (Springenberg et al., 2014), and each convolution, initialized using He normal initializer (He et al., 2015), is followed by a batch normalization, a rectified linear unit (ReLU) activation function, and a dropout layer with increasing fraction from $0.1-0.3$ in the encoding part, and vice versa in the decoding part of the network (Supplementary Figure 1). The network takes as input $3 \mathrm{D}$ volumes consisting of 16 neighboring slices for each of the three UTE images, the echo images and the derived $\mathrm{R}_{2}{ }^{*}$-map (16 slices $\times 192$ voxels $\times 192$ voxels $\times 3$ channels $)$, and outputs the corresponding CT slices ( 16 slices $\times 192$ voxels $\times 192$ voxels $\times 1$ channel). We used the HU-converted co-registered CT image as our target. We trained the 3D-network in Keras (Chollet, 2015) with TensorFlow backend (Abadi et al., 2016) using the Adam optimizer (learning rate $\left.=10^{-4}\right)($ Kingma and $\mathrm{Ba}, 2014)$, meansquared-error as loss function, batch size of 2 for 100 epochs. The 35 million parameters that were determined during the training process took 2 days on a Titan V (NVIDIA Corporation, Santa Clara, CA, United States) graphics processing unit. From our cohort of 79 scans, we did a 4 -fold cross validation, effectively training 4 networks on approximately 60 scans and evaluation on the remaining. During testing, we predicted the 3D pseudo-CT volumes around each slice, and computed the average voxel value for each of the overlapping volumes.

Since the CT coverage were usually less than the PET/MRI coverage, we added the DIXON-AC attenuation map outside the CT field-of-view. This was also done for the subsequently generated RESOLUTE and DeepUTE attenuation maps to allow for a fair comparison to the reference.

\section{Image Processing and Analysis}

Image processing and analysis were performed similar to our previous analysis of adult post-operative brain tumor FETPET patients (Ladefoged et al., 2017). First, a background (B) region of interest was delineated in healthy appearing gray and white matter at a level above the insula in the contralateral hemisphere to the tumor. The biological tumor volume (BTV) of FET-PET was measured using a 3D auto-contour using Mirada XD software (Mirada Medical, Oxford, United Kingdom) defining tumor tissue at a threshold above 1.6 of the mean standardized uptake value (SUV) in the background ROI (Floeth et al., 2005) for each AC method separately. Extratumoral areas with high FET uptake, e.g., vascular structures, pineal body and skin, were identified on either the T1w or FETPET image and removed from evaluation. The delineation was performed by a nuclear medicine specialist experienced in pediatric neurooncology (LM).

We assessed the different AC methods ability to produce accurate FET-PET images on a patient-by-patient basis using the most commonly semi-quantitative clinical metrics in the diagnostic workflow. We measured the biological tumor volume (BTV), mean ( $\mathrm{T}_{\text {MEAN }}$ ) and max $\left(\mathrm{T}_{\text {MAX }}\right)$, and the ratios $\mathrm{T}_{\text {MEAN }} / \mathrm{B}$ and $\mathrm{T}_{\text {MAX }} / \mathrm{B}$ were calculated. For the BTV we analyzed the tumor contours relative to the CT-AC reference using the Jaccard similarity metric, and a measurement of shape deviations. The calculated ratios were compared to the ratios calculated with the reference CT-AC. These metrics are commonly used as a criterion to identify active tumor tissue from reactive changes. As described previously (Ladefoged et al., 2017), we defined acceptance criteria of $< \pm 0.05$ and 0.1 or $5 \%$ for the $\mathrm{T}_{\mathrm{MEAN}} / \mathrm{B}$ and $\mathrm{T}_{\mathrm{MAX}} / \mathrm{B}$ ratios, respectively, and $\pm 2 \mathrm{~mL}$ or $10 \%$ for the BTV. These were based on differences in clinical practice that may be considered clinically relevant in identifying biologically active tumor tissue or treatment related change in activity (Piroth et al., 2011). The mix of both an absolute and relative cutoff reflects that larger absolute change is acceptable in large or very active tumors. For each clinical metric we calculated the mean difference, 95\% confidence intervals (CI) and limits of agreement on the log-transformed data, as the data was found to have log normal distribution. Exponentiation was applied to 
these results to express the differences as ratios on the original scale and report them as percentage differences. We corrected for repeated measurements from the repeated examinations (Bland and Altman, 1999).

\section{RESULTS}

A total of 28 patients met the inclusion criteria of $1 \mathrm{~mL}$ active tumor area, 6 of which had one or more follow up examination, resulting in a total of 36 examinations used for evaluation (Supplementary Table 1). Both RESOLUTE and DeepUTE were able to derive attenuation maps for all pediatric patients regardless of the age. Ten of the 28 patients (35\%) had titanium implants present. Overall, DeepUTE had improved accuracy over RESOLUTE: the Jaccard index was 0.57/0.62 in air, $0.74 / 0.79$ in soft tissue and $0.53 / 0.70$ in bone tissue for RESOLUTE/DeepUTE, respectively. The improved accuracy was also apparent in a direct visual comparison of the estimation of regional attenuation values in the nasal cavities, the skull base and the mastoid processes, and can be appreciated in Figure 1, where two patients with challenging anatomy are shown for RESOLUTE-AC, DeepUTE-AC and CT-AC, and the relative difference PET image in Supplementary Figure 2. Another example of a typical patient is given in Figure 2. There was also a significant improvement in AC runtime with values of $4 \mathrm{~s}$ for DeepUTE and $\sim 3$ min for of RESOLUTE, which although small, improves the overall imaging workflow.

Across all pediatric patients, the Jaccard index of the tumor delineation was $0.73 \pm 0.20$ for DIXON-AC, $0.90 \pm 0.07$ for RESOLUTE and $0.92 \pm 0.07$ for DeepUTE. The tumor configuration did not change for any of the patients when using RESOLUTE or DeepUTE compared to CT-AC but for DIXON$\mathrm{AC}$ this was found in 4 examinations (mean difference: $1.6 \mathrm{~mL}$ ), and was completely missed for an additional examination (BTV with CT-AC: $2 \mathrm{~mL}$ ).

The comparison of the clinical metrics can be seen in Figure 3, together with the defined acceptable limits. Across all metrics, using DeepUTE, none of the patients were outside the acceptable limits, whereas two patients fall short of the $\mathrm{T}_{\mathrm{MAX}} / \mathrm{B}$ limit and a single patient in the $\mathrm{T}_{\text {MEAN }}$ / B limit when using RESOLUTE. In these patients, the largest difference was $\mathrm{T}_{\mathrm{MAX}} / \mathrm{B}$ overestimation

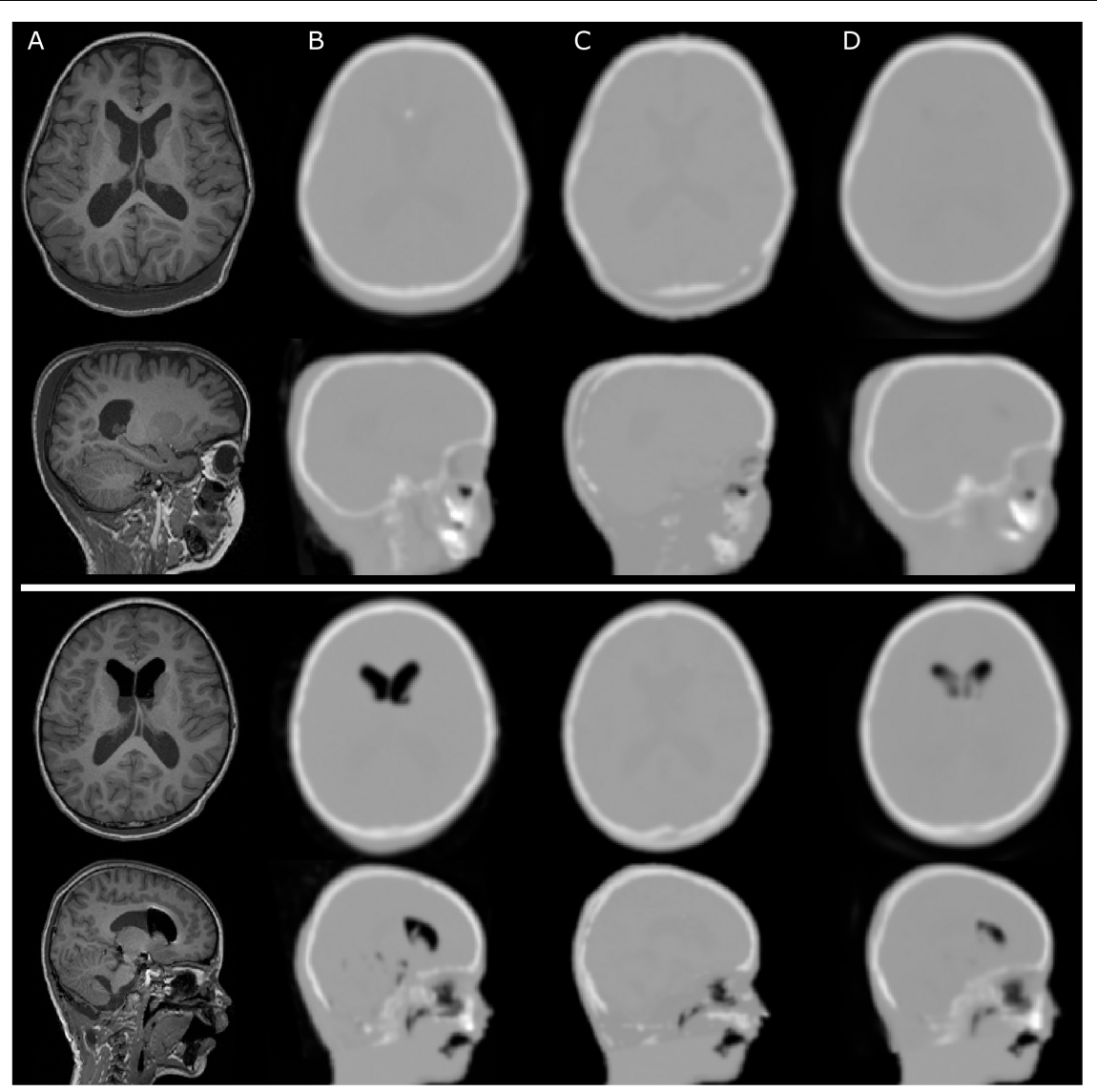

FIGURE 1 | Sample cases for two pediatric patients with irregular anatomy. (A) show the T1w MPRAGE, (B) CT-AC, (C) RESOLUTE-AC, and (D) DeepUTE-AC. The top rows show a 5-year-old patient with post-operative subcutaneous soft tissue swelling in the occipital region. RESOLUTE erroneously fills in a dual layer bone layer on both sides of the swelling, along skin and bone. The bottom rows show a 6-year-old patient with air pockets anteriorly in the lateral ventricles that appeared after surgical intervention, and are not imaged in RESOLUTE. Also in this case RESOLUTE crafts a dual layered skull in the occipital region. For both patients, RESOLUTE is challenged in the definition of facial and skull base attenuation value. DeepUTE captures the morphology more confidently. 


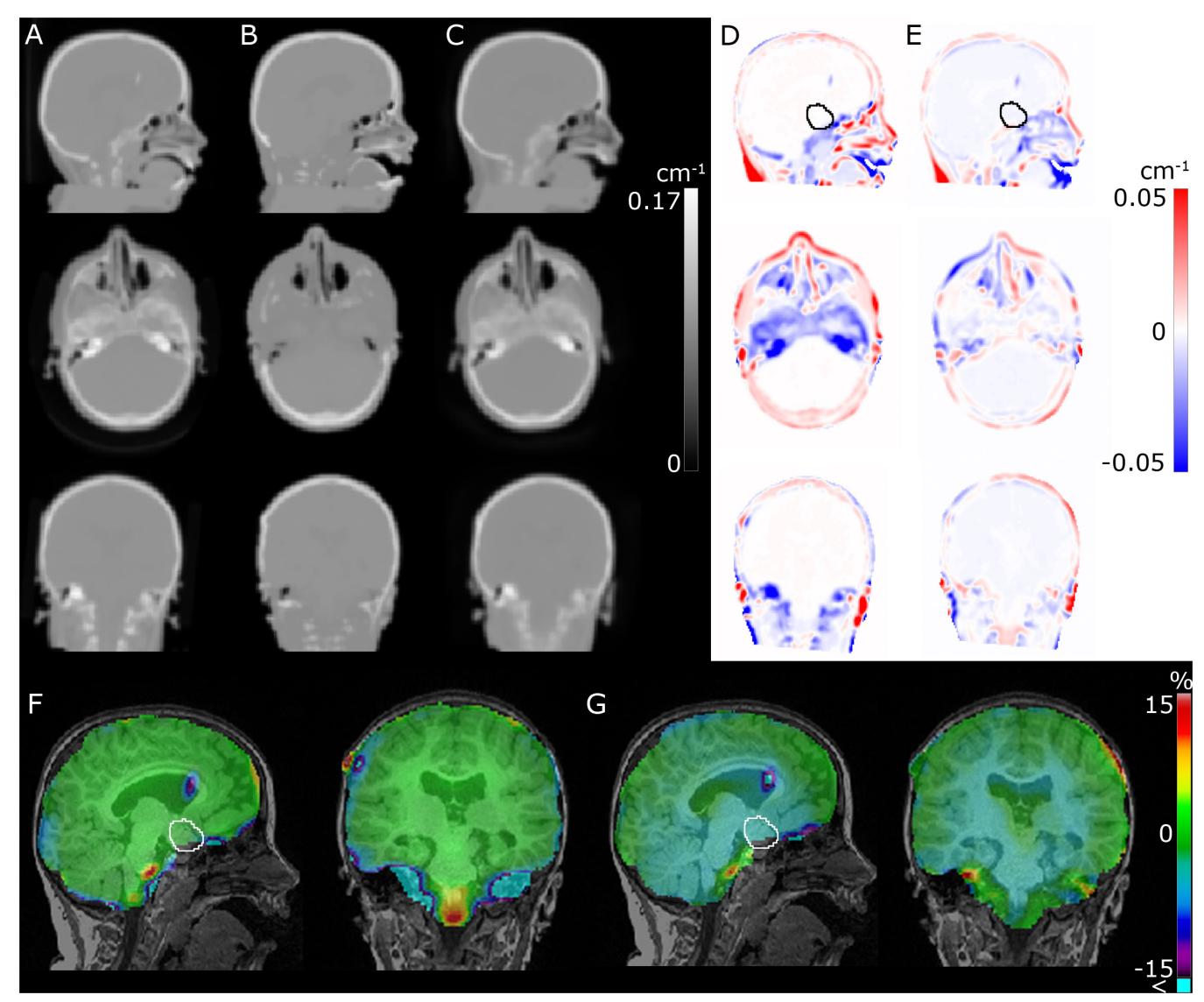

FIGURE 2 | Comparison of CT (A), RESOLUTE (B), and DeepUTE (C) attenuation maps in the sagittal, axial and coronal orientation, respectively. (D,E) shows (B,C) subtracted $(\mathbf{A})$, respectively, and $\mathbf{( F , G )}$ shows the resulting relative difference in the PET images between RESOLUTE and DeepUTE relative to CT-AC, respectively. The improved accuracy in the nasal cavities, the skull base and the mastoid processes, leads to a clear reduction of the errors in the surrounding regions, e.g., in the medulla. It also appears that, for this patient, a small underestimation of the densities within the brain in DeepUTE leads to a small underestimation globally within the brain. The tumor delineation is show on the sagittal view in (D-G).

of 0.13 a.u. due to overestimated bone area in the skull base. In comparison, DIXON-AC gave a $\mathrm{T}_{\mathrm{MAX}} / \mathrm{B}$ difference over the acceptable limit in 23/36 (64\%) examinations, and 13/36 (36\%) examinations had changes to BTV over the acceptable limit.

The relative \%-difference in the diagnostic measures was similar between RESOLUTE and DeepUTE, again with DeepUTE with the reduced error and variation (Table 1). BTV measured using DeepUTE was underestimated by $2 \%$ on average $(95 \%$ CI: -5 to $1 \%)$ compared to $-1 \%(95 \% \mathrm{CI}:-5$ to $4 \%)$ with RESOLUTE. None of the metrics had statistically significant differences compared to the reference CT-AC. In comparison, DIXON-AC had statistically significant differences in all three clinical metrics $(p<0.001)$.

\section{DISCUSSION}

Magnetic resonance imaging is the method of choice to diagnose brain tumor patients, but FET-PET can add valuable information for clinical decision making (Dunkl et al., 2015). Examining pediatric and adolescent patients on a hybrid PET/MRI can be preferred over PET/CT to reduce the number of examinations, which is especially relevant when anesthesia is required, and is important for both child and parents. A prerequisite for a confident clinical evaluation of the cohort with PET/MRI is an accurate AC. The skull shape, density, thickness, and composition change considerably during development in childhood especially the first three years after which the sutures and fontanelles gradually calcify and close (Li et al., 2015). Especially the rapid growth of skull thickness and bone density will highly influence attenuation leading to errors in atlas-based methods that cannot account for the thin, low-density infant cranium.

In designing the clinical study, we were acutely aware of these unresolved AC issues and choose to include a separate low-dose CT acquisition. This could be performed safely in all children, although it involved moving sensitive patients to a different scanner for additional radiation exposure and, for some children, extending anesthesia. This additional stress on the patients was regarded ethically acceptable so that future use of hybrid PET/MRI in pediatric brain tumors, which could be one of the most important applications, could be performed with the best possible assessment of risk to the patient caused by 


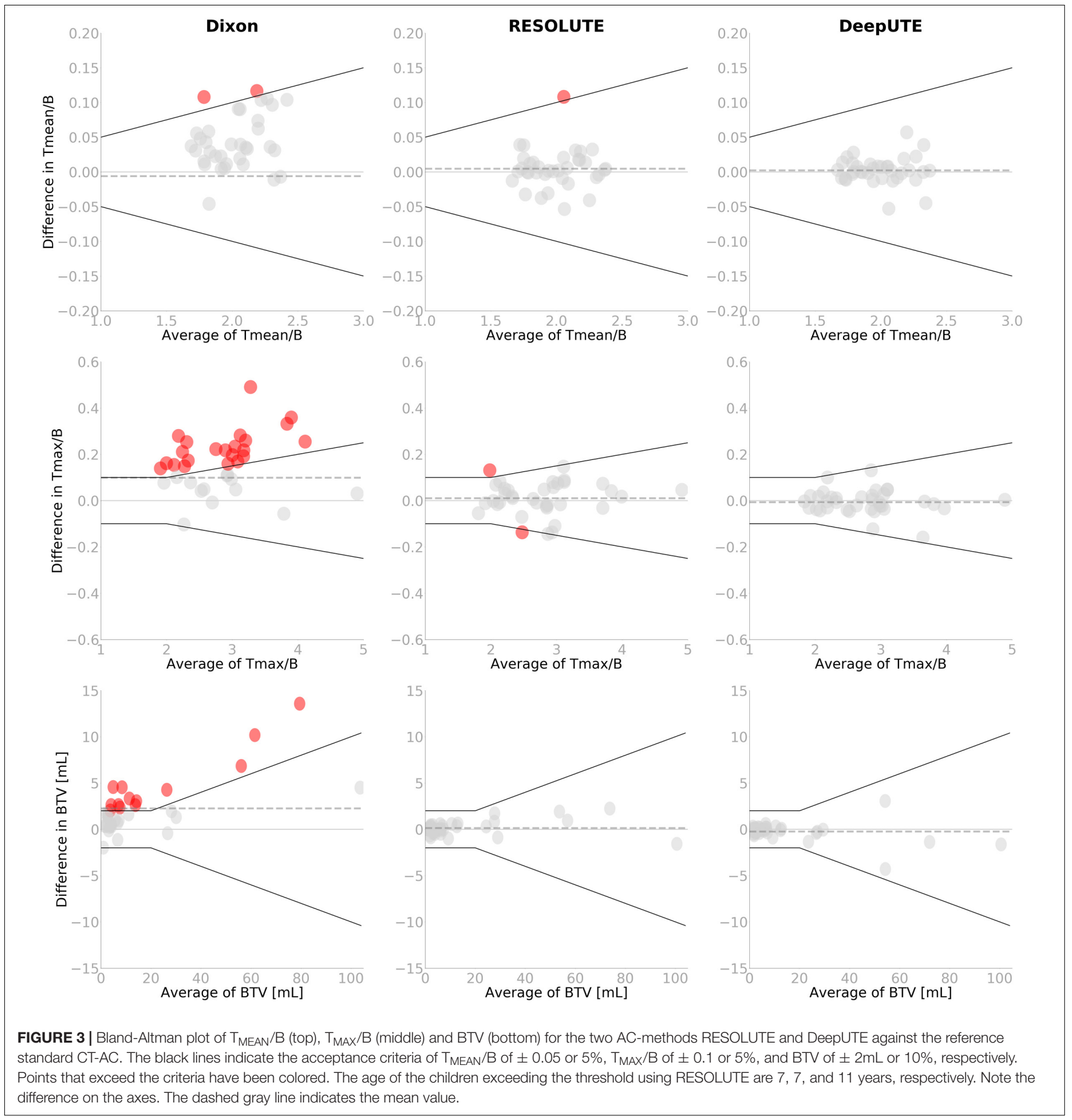

quantitative inaccuracies using accepted standard metrics within the field.

We modified the already thoroughly evaluated RESOLUTE method to be applied on pediatric patients, as well as introduced an MR-AC method based on a deep learning convolutional neural network, and also included DIXON-AC. The novelty of DeepUTE does not lie in the chosen type of architecture, but rather in the data that went into training the model. This manuscript is, to the best of our knowledge, the first of its kind to train a deep learning network for MR-AC purposes on a pediatric cohort of this size. The included patients in the evaluated cohort are well suited to test the method's ability to adapt to anatomy changes across different ages.

Pediatric patients are a challenging cohort to examine due to motion, often leading to sedation or anesthesia of the patients. The patients included in this study had, as expected, a larger amount of noise in the MR images than adult patients, leading to increased amount of noise in the bone surrogate signal. The 
TABLE 1 | Summary of the relative \%-difference* to the reference CT-AC of each clinical metric for the MR-AC methods.

\begin{tabular}{|c|c|c|c|c|c|}
\hline \multirow{2}{*}{$\begin{array}{l}\text { Measured } \\
\text { parameter } \\
\text { values }\end{array}$} & \multicolumn{3}{|c|}{ Mean $\%$ difference } & \multirow{2}{*}{$\begin{array}{l}95 \% \text { lower } \\
\text { limits of } \\
\text { agreement }\end{array}$} & \multirow{2}{*}{$\begin{array}{l}95 \% \text { upper } \\
\text { limits of } \\
\text { agreement }\end{array}$} \\
\hline & Mean & $95 \% \mathrm{Cl}$ & $p$ & & \\
\hline \multicolumn{6}{|l|}{ DIXON-AC } \\
\hline $\mathrm{T}_{\mathrm{MEAN}} / \mathrm{B}$ & 2.2 & 1.5 to 2.8 & $<0.001^{* *}$ & -1.6 & 6.0 \\
\hline $\mathrm{T}_{\mathrm{MAX}} / \mathrm{B}$ & 5.9 & 4.5 to 7.4 & $<0.001^{* *}$ & -2.2 & 14.7 \\
\hline BTV & 32 & 21 to 45 & $<0.001^{* *}$ & -22 & 124 \\
\hline \multicolumn{6}{|c|}{ RESOLUTE } \\
\hline $\mathrm{T}_{\mathrm{MEAN}} / \mathrm{B}$ & 0.2 & -0.3 to 0.7 & 0.38 & -2.6 & 3.1 \\
\hline $\mathrm{T}_{\text {MAX }} / \mathrm{B}$ & 0.3 & -0.6 to 1.2 & 0.54 & -4.9 & 5.8 \\
\hline BTV & -1 & -5 to 4 & 0.77 & -21 & 25 \\
\hline \multicolumn{6}{|l|}{ DeepUTE } \\
\hline $\mathrm{T}_{\mathrm{MEAN}} / \mathrm{B}$ & -0.1 & -0.2 to 0.5 & 0.38 & -1.7 & 2.0 \\
\hline $\mathrm{T}_{\mathrm{MAX}} / \mathrm{B}$ & -0.1 & -0.8 to 0.5 & 0.67 & -3.7 & 3.6 \\
\hline BTV & -2 & -5 to 1 & 0.15 & -19 & 17 \\
\hline
\end{tabular}

* Exponentiation was applied to results from analysis on log scale, and results were expressed as percentages. ${ }^{* *}$ Indicates a statistical significant $(p<0.05)$ found by a paired t-test. $\mathrm{Cl}=95 \%$ confidence interval for mean difference. BTV is measured in $m L$. A single examination without BTV with DIXON-AC was left out of this analysis.

strength of the DeepUTE method is that it is able to robustly handle this noise, which the deep learning methods are known for. An example of the improved noise handling is evident in Figure 1, where DeepUTE better models both the thin bone and noise at the posterior part of the head.

Titanium alloy clamps, that were present in $33 \%$ of the patients to fix the craniotomy, showed up as small signal voids in the MR images with a size similar to the implants seen on CT. Visual reading showed that both RESOLUTE and DeepUTE filled the signal void with a density similar to dense bone, similar to what has previously been observed (Ladefoged et al., 2017). This meant that a valid attenuation map without artifacts could be calculated in all scans using RESOLUTE and DeepUTE.

Overall, we found both RESOLUTE and our DeepUTE methodology to accurately reproduce the CT-AC clinical metrics with similar accuracy as was seen for RESOLUTE when evaluating adult FET-PET brain tumor patients (Ladefoged et al., 2017). Regardless of age, both methods were able to obtain AC maps similar to the CT-AC, albeit with DeepUTE producing the most similar based on both quantitative metrics and visual inspection. In the patient-by-patient analysis, all patients were inside the predefined acceptable clinical limits with DeepUTE, where three patients (7-11 years old) were outside the limits in the $\mathrm{T}_{\text {MAX }} / \mathrm{B}$ or $\mathrm{T}_{\text {MEAN }} / \mathrm{B}$ metrics when using RESOLUTE (Figure 3). A similar result was obtained with RESOLUTE for the adult FET-PET brain tumor patients (Ladefoged et al., 2017) where 5/68 studies exceeded the predefined limit. The errors from RESOLUTE were due to an overestimation of bone density in known "problem" areas near the skull base, but none of the errors impacted the clinical reading of the images. In comparison, the same patients obtained with DeepUTE-AC had a higher precision in the skull base, leading to more accurate measurements. The confidence interval was narrower when using DeepUTE compared to RESOLUTE (Table 1). This indicates that there is a smaller variation of the errors in DeepUTE compared to RESOLUTE.

The processing in RESOLUTE was the same for all patients, except for the combination of the segmented tissue maps within regional masks, as these are different depending on the patient age. In DeepUTE, the same method was applied regardless of patient age. Further dividing the training patients into smaller groups depending on age might further reduce the variance, but requires more data, as training a deep learning network with too few patients leads to overfitting. We did not apply transfer learning in this study, as it has been shown that training a deep learning network using less than 30 patients is feasible (Han, 2017; Liu et al., 2017; Gong et al., 2018; Kläser et al., 2018; Leynes et al., 2018). However, using transfer learning, e.g., from a larger adult cohort might further improve the results presented here, as the low-level information are to be expected similar between the cohorts.

In software version VB20P on the Siemens mMR, two vendor-provided solutions for AC is available - DIXON-AC and UTE-AC, that both have been used in the published pediatric neuro-oncology PET/MRI literature (Garibotto et al., 2013; Preuss et al., 2014; Fraioli et al., 2015), however, encompassing only 6 and 12 patients, respectively. This small patient sample may reflect hesitation from the clinical community to use PET/MRI routinely in this difficult patient group because of the well-documented systematic underperformance of particularly DIXON-AC (Andersen et al., 2014; Ladefoged et al., 2017), which is also apparent from our study. DIXON-AC was the only vendorprovided method capable of producing attenuation maps for the full pediatric cohort. In four patients, UTE-AC was not able to produce an attenuation map of patients, aged $0-2$ years, which is why we chose to exclude UTE-AC from the comparison.

In this study, we only had 6 patients with repeat examinations. We found that the change of $\mathrm{T}_{\text {MEAN }} / \mathrm{B}, \mathrm{T}_{\mathrm{MAX}} / \mathrm{B}$ and $\mathrm{BTV}$ between two examinations with RESOLUTE or DeepUTE were in congruence with the change when measured with CT-AC, as none of the differences were outside the acceptable limit. A larger number of repeat examinations should confirm this.

\section{Limitations}

We did not have pediatric data available after the software upgrade to VE11P, which adds a model-based AC method (Paulus et al., 2015; Koesters et al., 2016), but we speculate that the method would be unsuccessful for the younger pediatric cohort since the method was developed for adults.

Both RESOLUTE and DeepUTE are based on the UTE sequence, so while we expect DeepUTE to be directly transferable to any Siemens mMR, which is the case for RESOLUTE, neither method is able to produce attenuation maps from PET/MRI data from other vendors. The fundamental idea behind DeepUTE is not limited to UTE data, and retraining the network on other MR sequences such as the T1w MPRAGE or ZTE could allow for a multi-vendor method. However, it would require a large pediatric dataset across several vendors to confirm this.

Although, the limits of agreement using RESOLUTE and DeepUTE are encouragingly narrow (Table 1), the number of patients in each age category is still small. Thus, we cannot 
rule out artifacts caused by other combinations of anatomy and pathology.

\section{CONCLUSION}

The present study performed on FET-PET/MRI examinations of pediatric patients revealed that both RESOLUTE and our deep learning method DeepUTE are able to robustly produce attenuation maps similar to the reference CT-AC. The clinical metrics were within acceptable limits of the reference CT-AC, making either method suitable for imaging of pediatric brain tumor patients - a cohort that is especially challenging for atlasbased methods. For clinical use of any MR-AC map, however, we recommend visually inspection for artifacts with particular attention to areas close to the skull base, anatomically distorted tissue and metal implants. The added accuracy due to better noise handling of DeepUTE, ease of use without the need for regional masks, as well as the improved runtime makes DeepUTE the method of choice for PET/MRI AC. Further refinement of the deep learning method with age-specific data input is likely to improve the performance.

\section{AUTHOR CONTRIBUTIONS}

CL designed the method, did the data analysis, and prepared the manuscript. LM and IL designed the method, aided in data

\section{REFERENCES}

Abadi, M., Barham, P., Chen, J., Chen, Z., Davis, A., Dean, J., et al. (2016). "TensorFlow: a system for large-scale machine learning," in Proceedings of the Twelth USENIX Symposium on Operating Systems Design and Implementation, Savannah, GA, 265-283.

Andersen, F. L., Ladefoged, C. N., Beyer, T., Keller, S. H., Hansen, A. E., Højgaard, L., et al. (2014). Combined PET/MR imaging in neurology: MR-based attenuation correction implies a strong spatial bias when ignoring bone. Neuroimage 84, 206-216. doi: 10.1016/j.neuroimage.2013. 08.042

Bezrukov, I., Schmidt, H., Gatidis, S., Schwenzer, N., and Pichler, B. J. (2015). Quantitative evaluation of segmentation- and atlas-based attenuation correction for PET/MR on pediatric patients. J. Nucl. Med. 56, 1067-1075. doi: 10.2967/jnumed.114.149476

Bland, J. M., and Altman, D. G. (1999). Measuring agreement in method comparison studies. Stat. Methods Med. Res. 8, 135-160. doi: 10.1177/ 096228029900800204

Buchmann, N., Klasner, B., Gempt, J., Bauer, J. S., Pyka, T., Delbridge, C., et al. (2016). (18)F-fluoroethyl-l-thyrosine positron emission tomography to delineate tumor residuals after glioblastoma resection: a comparison with standard postoperative magnetic resonance imaging. World Neurosurg. 89, 420-426. doi: 10.1016/j.wneu.2016.02.032

Çiçek, Ö, Abdulkadir, A., Lienkamp, S. S., Brox, T., and Ronneberger, O. (2016). "3D U-Net: learning dense volumetric segmentation from sparse annotation," in Proceedings of the International Conference on Medical Image Computing and Computer-Assisted Intervention, (Berlin: Springer International Publishing), 424-432. doi: 10.1007/978-3-319-46723-8_49

Chollet, F. (2015). Keras. Available at: https://github.com/fchollet/keras

Delso, G., Fürst, S., Jakoby, B., Ladebeck, R., Ganter, C., Nekolla, S. G., et al. (2011). Performance measurements of the Siemens mMR integrated wholebody PET/MR scanner. J. Nucl. Med. 52, 1914-1922. doi: 10.2967/jnumed.111. 092726 analysis, and revised and approved the manuscript. AH, FA, and $\mathrm{LH}$ aided in data acquisition, and revised and approved the manuscript.

\section{FUNDING}

The generous support by The Danish Childhood Cancer Foundation is highly appreciated (2014-34, 2015-48).

\section{ACKNOWLEDGMENTS}

We highly appreciate the scanner assistance of technologists Karin Stahr and Marianne Federspiel, and of radiographers Jákup Martin Poulsen and Nadia Azizi. The John and Birthe Meyer Foundation is thanked for their generous donation of the Siemens mMR hybrid PET/MR system at Copenhagen University Hospital Rigshospitalet.

\section{SUPPLEMENTARY MATERIAL}

The Supplementary Material for this article can be found online at: https://www.frontiersin.org/articles/10.3389/fnins. 2018.01005/full\#supplementary-material

Dunkl, V., Cleff, C., Stoffels, G., Judov, N., Sarikaya-Seiwert, S., Law, I., et al. (2015). The usefulness of dynamic O-(2-[18F]fluoroethyl)-L-tyrosine-PET in the clinical evaluation of brain tumors in children and adolescents. J. Nucl. Med. 56, 88-92. doi: 10.2967/jnumed.114.148734

Floeth, F. W., Pauleit, D., Wittsack, H. J., Langen, K. J., Reifenberger, G., Hamacher, K., et al. (2005). Multimodal metabolic imaging of cerebral gliomas: positron emission tomography with $[18 \mathrm{~F}]$ fluoroethyl-L-tyrosine and magnetic resonance spectroscopy. J. Neurosurg. 102, 318-327. doi: 10.3171/jns.2005.102. 2.0318

Fonov, V., Evans, A. C., Botteron, K., Almli, C. R., McKinstry, R. C., Collins, D. L., et al. (2011). Unbiased average age-appropriate atlases for pediatric studies. Neuroimage 54, 313-327. doi: 10.1016/j.neuroimage.2010.07.033

Fraioli, F., Shankar, A., Hargrave, D., Hyare, H., Gaze, M. N., Groves, A. M., et al. (2015). 18F-fluoroethylcholine (18F-Cho) PET/MRI functional parameters in pediatric astrocytic brain tumors. Clin. Nucl. Med. 40, e40-e45. doi: 10.1097/ RLU.0000000000000556

Galldiks, N., Dunkl, V., Stoffels, G., Hutterer, M., Rapp, M., Sabel, M., et al. (2015a). Diagnosis of pseudoprogression in patients with glioblastoma using O-(2-[18F]fluoroethyl)-L-tyrosine PET. Eur. J. Nucl. Med. Mol. Imaging 42, 685-695. doi: 10.1007/s00259-014-2959-4

Galldiks, N., Stoffels, G., Filss, C., Rapp, M., Blau, T., Tscherpel, C., et al. (2015b). The use of dynamic O-(2-18F-fluoroethyl)-1-tyrosine PET in the diagnosis of patients with progressive and recurrent glioma. Neuro Oncol. 17, 1293-1300. doi: 10.1093/neuonc/nov088

Garibotto, V., Heinzer, S., Vulliemoz, S., Guignard, R., Wissmeyer, M., Seeck, M., et al. (2013). Clinical applications of hybrid PET/MRI in neuroimaging. Clin. Nucl. Med. 38, e13-e18. doi: 10.1097/RLU.0b013e3182638ea6

Gong, K., Yang, J., Kim, K., El, Fakhri G, Seo, Y., and Li, Q. (2018). Attenuation correction for brain PET imaging using deep neural network based on dixon and ZTE MR images. Phys. Med. Biol. 63:125011. doi: 10.1088/1361-6560/ aac763

Han, X. (2017). MR-based synthetic CT generation using a deep convolutional neural network method. Med. Phys. 44, 1408-1419. doi: 10.1002/mp.12155 
He, K., Zhang, X., Ren, S., and Sun, J. (2015). “Delving deep into rectifiers: Surpassing human-level performance on imagenet classification," in Proceedings of the IEEE International Conference on Computer Vision, Nice, 1026-1034. doi: 10.1109/ICCV.2015.123

Henriksen, O. M., Marner, L., and Law, I. (2016). Clinical PET/MR imaging in dementia and neuro-oncology. PET Clin. 11, 441-452. doi: 10.1016/j.cpet.2016. 05.003

Jakoby, B. W., Bercier, Y., Watson, C. C., Bendriem, B., and Townsend, D. W. (2009). Performance characteristics of a new LSO PET/CT scanner with extended axial field-of-view and PSF reconstruction. IEEE Trans. Nucl. Sci. 56, 633-639. doi: 10.1109/TNS.2009.2015764

Juttukonda, M. R., Mersereau, B. G., Chen, Y., Su, Y., Rubin, B. G., Benzinger, T. L. S., et al. (2015). MR-based attenuation correction for PET/MRI neurological studies with continuous-valued attenuation coefficients for bone through a conversion from R2* to CT-Hounsfield units. Neuroimage 112, 160-168. doi: 10.1016/j.neuroimage.2015.03.009

Kingma, D. P., and Ba, J. (2014). Adam: a method for stochastic optimization. arXiv [Preprint]. arXiv:1412.6980

Kläser, K., Markiewicz, P., Ranzini, M., Li, W., Modat, M., Hutton, B. F., et al. (2018). International Workshop on Simulation and Synthesis in Medical Imaging. Cham: Springer, 61-70.

Koesters, T., Friedman, K. P., Fenchel, M., Zhan, Y., Hermosillo, G., Babb, J., et al. (2016). Dixon sequence with superimposed model-based bone compartment provides highly accurate PET/MR attenuation correction of the brain. J. Nucl. Med. 57, 918-924. doi: 10.2967/jnumed.115.166967

Kracht, L. W., Miletic, H., Busch, S., Jacobs, A. H., Voges, J., Hoevels, M., et al. (2004). Delineation of brain tumor extent with [11C]L-methionine positron emission tomography: local comparison with stereotactic histopathology. Clin. Cancer Res. 10, 7163-7170. doi: 10.1158/1078-0432.CCR-04-0262

Ladefoged, C. N., Andersen, F. L., Kjær, A., Højgaard, L., and Law, I. (2017). RESOLUTE PET/MRI attenuation correction for O-(2-18F-fluoroethyl)-Ltyrosine (FET) in brain tumor patients with metal implants. Front. Neurosci. 11:453. doi: 10.3389/fnins.2017.00453

Ladefoged, C. N., Benoit, D., Law, I., Holm, S., Kjær, A., Højgaard, L., et al. (2015). Region specific optimization of continuous linear attenuation coefficients based on UTE (RESOLUTE): application to PET/MR brain imaging. Phys. Med. Biol. 60, 8047-8065. doi: 10.1088/0031-9155/60/20/8047

Ladefoged, C. N., Law, I., Anazodo, U., St. Lawrence, K., Izquierdo-Garcia, D., Catana, C., et al. (2016). A multi-centre evaluation of eleven clinically feasible brain PET/MRI attenuation correction techniques using a large cohort of patients. Neuroimage 147, 346-359. doi: 10.1016/j.neuroimage.2016.12.010

Langen, K. J., Hamacher, K., Weckesser, M., Floeth, F., Stoffels, G., Bauer, D., et al. (2006). O-(2-[18F]fluoroethyl)-l-tyrosine: uptake mechanisms and clinical applications. Nucl. Med. Biol. 33, 287-294. doi: 10.1016/j.nucmedbio.2006. 01.002

Leynes, A. P., Yang, J., Wiesinger, F., Kaushik, S. S., Shanbhag, D. D., Seo, Y., et al. (2018). Zero-echo-time and dixon deep pseudo-CT (ZeDD CT): direct generation of pseudo-CT images for pelvic PET/MRI attenuation correction using deep convolutional neural networks with multiparametric MRI. J. Nucl. Med. 59, 852-858. doi: 10.2967/jnumed.117.198051

Li, Z., Park, B. K., Liu, W., Zhang, J., Reed, M. P., Rupp, J. D., et al. (2015). A statistical skull geometry model for children 0-3 years old. PLoS One 10:e0127322. doi: 10.1371/journal.pone.0127322

Liu, F., Jang, H., Kijowski, R., Bradshaw, T., and McMillan, A. B. (2017). Deep learning MR imaging-based attenuation correction for PET/MR imaging. Radiology 286, 676-684. doi: 10.1148/radiol.2017170700
Martinez-Möller, A., Souvatzoglou, M., Delso, G., Bundschuh, R. A., Chefd'hotel, C., Ziegler, S. I., et al. (2009). Tissue classification as a potential approach for attenuation correction in whole-body PET/MRI: evaluation with PET/CT data. J. Nucl. Med. 50, 520-526. doi: 10.2967/jnumed.108. 054726

Mullins, M. E., Barest, G. D., Schaefer, P. W., Hochberg, F. H., Gonzalez, R. G., and Lev, M. H. (2005). Radiation necrosis versus glioma recurrence: conventional MR imaging clues to diagnosis. AJNR Am. J. Neuroradiol. 26, 1967-1972.

Paulus, D. H., Quick, H. H., Geppert, C., Fenchel, M., Hermosillo, G., Faul, D., et al. (2015). Whole-body PET/MR imaging: quantitative evaluation of a novel model-based MR attenuation correction method including bone. J. Nucl. Med. 56, 1061-1066. doi: 10.2967/jnumed.115.156000

Piroth, M. D., Pinkawa, M., Holy, R., Klotz, J., Nussen, S., Stoffels, G., et al. (2011). Prognostic value of early [18F]fluoroethyltyrosine positron emission tomography after radiochemotherapy in glioblastoma multiforme. Int. J. Radiat. Oncol. 80, 176-184. doi: 10.1016/j.ijrobp.2010.01.055

Preuss, M., Werner, P., Barthel, H., Nestler, U., Christiansen, H., Hirsch, F. W., et al. (2014). Integrated PET/MRI for planning navigated biopsies in pediatric brain tumors. Child's Nerv. Syst. 30, 1399-1403. doi: 10.1007/s00381-0142412-9

Robson, M. D., Gatehouse, P. D., Bydder, M., and Bydder, G. M. (2003). Magnetic resonance: an introduction to ultrashort TE (UTE) imaging. J. Comput. Assist. Tomogr. 27, 825-846. doi: 10.1097/00004728-200311000-00001

Ronneberger, O., Fischer, P., and Brox, T. (2015). U-Net: convolutional networks for biomedical image segmentation. Med Image Comput. Comput. Interv. 9351, 234-241. doi: 10.1007/978-3-319-24574-4_28

Sekine, T., Voert, E. E. G. W., Warnock, G., Buck, A., Huellner, M., Veit-haibach, P., et al. (2016). Clinical evaluation of zero-echo-time attenuation correction for brain 18F-FDG PET/MRI: comparison with atlas attenuation correction. J. Nucl. Med. 57, 1927-1932. doi: 10.2967/jnumed.116. 175398

Spick, C., Herrmann, K., and Czernin, J. (2016). PET/CT and PET/mri perform equally well in cancer: evidence from studies on more Than 2,300 patients. J. Nucl. Med. 57, 420-431. doi: 10.2967/jnumed.115.158808

Springenberg, J. T., Dosovitskiy, A., Brox, T., and Riedmiller, M. (2014). Striving for simplicity: the all convolutional net. arXiv [Preprint]. arXiv:1412.6806

Vander Borght, T., Asenbaum, S., Bartenstein, P., Halldin, C., Kapucu, O., Van Laere, K., et al. (2006). EANM procedure guidelines for brain tumour imaging using labelled amino acid analogues. Eur. J. Nucl. Med. Mol. Imaging 33, 1374-1380. doi: 10.1007/s00259-006-0206-3

Watanabe, M., Tanaka, R., and Takeda, N. (1992). Magnetic resonance imaging and histopathology of cerebral gliomas. Neuroradiology 34, 463-469. doi: 10.1007/ BF00598951

Conflict of Interest Statement: The authors declare that the research was conducted in the absence of any commercial or financial relationships that could be construed as a potential conflict of interest.

Copyright (c) 2019 Ladefoged, Marner, Hindsholm, Law, Højgaard and Andersen. This is an open-access article distributed under the terms of the Creative Commons Attribution License (CC BY). The use, distribution or reproduction in other forums is permitted, provided the original author(s) and the copyright owner(s) are credited and that the original publication in this journal is cited, in accordance with accepted academic practice. No use, distribution or reproduction is permitted which does not comply with these terms. 